\title{
Stability of the Electroweak Vacuum: Gauge Independence and Advanced Precision
}

\author{
A. V. Bednyakov, ${ }^{1}$ B. A. Kniehl, ${ }^{2}$ A. F. Pikelner, ${ }^{2}$ and O. L. Veretin ${ }^{2}$ \\ ${ }^{1}$ Joint Institute for Nuclear Research, 141980 Dubna, Russia \\ ${ }^{2}$ II. Institut für Theoretische Physik, Universität Hamburg, Luruper Chaussee 149, 22761 Hamburg, Germany
}

(Received 30 July 2015; revised manuscript received 24 August 2015; published 9 November 2015)

\begin{abstract}
We perform a manifestly gauge-independent analysis of the vacuum stability in the standard model including two-loop matching, three-loop renormalization group evolution, and pure QCD corrections through four loops. All these ingredients are exact, except that light-fermion masses are neglected. We in turn apply the criterion of nullifying the Higgs self-coupling and its beta function in the modified minimalsubtraction scheme and a recently proposed consistent method for determining the true minimum of the effective Higgs potential that also avoids gauge dependence. Exploiting our knowledge of the Higgs-boson mass, we derive an upper bound on the pole mass of the top quark by requiring that the standard model be stable all the way up to the Planck mass scale and conservatively estimate the theoretical uncertainty. This bound is compatible with the Monte Carlo mass quoted by the Particle Data Group at the $1.3 \sigma$ level.
\end{abstract}

The standard model (SM) of elementary particle physics has been enormously consolidated by the discovery [1] at the CERN Large Hadron Collider of a new weak neutral resonance that, within the present experimental uncertainty, shares the spin $(J)$, parity $(P)$, and charge-conjugation $(C)$ quantum numbers $J^{\mathrm{PC}}=0^{++}$and the coupling strengths with the SM Higgs boson $H$, in the absence of convincing signals of new physics beyond the SM. Moreover, its mass of $(125.7 \pm 0.4) \mathrm{GeV}$ [2] falls well inside the $M_{H}$ range predicted within the SM through global analyses of electroweak (EW) precision data [2]. Besides completing the SM particle multiplet and confirming the Higgs mechanism of mass generation via the spontaneous breaking of the EW symmetry proposed by Englert, Higgs (The Nobel Prize in Physics, 2013), and Brout, this groundbreaking discovery also has fundamental cosmological consequences by allowing conclusions regarding the fate of the Universe via the analysis of the vacuum stability [3]. In fact, owing to an intriguing conspiracy of the SM particle masses, chances are that the Higgs potential develops a second minimum, as deep as the one corresponding to the vacuum with expectation value (VEV) $v=2^{-1 / 4} G_{F}^{-1 / 2}=246 \mathrm{GeV}$ in which we live, at a field value of the order of the Planck mass $M_{P}=$ $1.22 \times 10^{19} \mathrm{GeV}[4,5]$. This would imply that the SM be stable all the way up to the energy scale where the unification with gravity is expected to take place anyways, which would diminish the necessity for grand unified theories at lower scales. EW symmetry breaking might thus be determined by Planck-scale physics [5], and the existence of a relationship between $M_{P}$ and SM parameters might signify a reduction of fundamental couplings. Of course, experimental facts that the SM fails to explain, such as the smallness of the neutrino masses, the strong $C P$ problem, the existence of dark matter, and the baryon asymmetry in the Universe, would then still call for an extension.
Obviously, the ultimate answer to the existential question whether our vacuum is stable or not crucially depends on the quality of the theoretical analysis as for both conceptual rigor and high precision, and it is the goal of this Letter to significantly push the state of the art by optimally exploiting information that has become available just recently. The technical procedure is as follows. The set of running coupling constants, including the $\mathrm{SU}(2)_{I}, U(1)_{Y}$, and $\mathrm{SU}(3)_{c}$ gauge couplings $g(\mu), g^{\prime}(\mu)$, and $g_{s}(\mu)$, respectively, the Higgs self-coupling $\lambda(\mu)$, and the Yukawa couplings $y_{f}(\mu)$, of the full SM are evolved in the renormalization scale $\mu$ from $\mu^{\text {thr }}=O(v)$ to $\mu^{\text {cri }}=$ $O\left(M_{P}\right)$ using the renormalization group (RG) equations. The beta functions appearing therein take a simple polynomial form in the modified minimal-subtraction ( $\overline{\mathrm{MS}}$ ) scheme of dimensional regularization. They are fully known through three loops [6] in the approximation of neglecting the Yukawa couplings of the first- and secondgeneration fermions, and the ones of $g_{s}$ [7] and $y_{q}$ [8] also at the four-loop order $O\left(\alpha_{s}^{4}\right)$, the latter being given by the quark mass anomalous dimension. The initial conditions at $\mu=\mu^{\text {thr }}$ are evaluated from the relevant constants of nature, including Sommerfeld's fine-structure constant $\alpha_{\mathrm{Th}}$ defined in Thomson scattering (or, alternatively, Fermi's constant $G_{F}$ ), the strong-coupling constant $\alpha_{S}^{(5)}\left(M_{Z}\right)$ at its reference point in QCD with $n_{f}=5$ active quark flavors, and the physical particle masses $M_{i}(i=W, Z, H, f)$ defined via the propagator poles, taking into account threshold corrections [9], which are fully known through two loops [5,10-14] and, for $g_{s}$ and $y_{q}$, also at $O\left(\alpha_{s}^{3}\right)[15,16]$ and even at $O\left(\alpha_{s}^{4}\right)[17,18]$. Although self-consistency requires that $n$-loop evolution is combined with $(n-1)$-loop matching, we, nevertheless, include the additional information $[17,18]$ in our default predictions. There are two approaches to the threshold corrections in the literature that 
differ in the definition of the $\overline{\mathrm{MS}} \mathrm{VEV} v(\mu)$. In the first one [10,11], $v(\mu)$ is fixed to be the minimum of the effective Higgs potential $V_{\text {eff }}(H)$ in the Landau gauge and is thus gauge dependent [19]. A solution to this problem has recently been proposed in Ref. [20]. In the second approach $[5,12-14]$, the adjustment of the VEV is only done for the bare theory, yielding $v^{0}=\sqrt{-\left(m_{\Phi}^{0}\right)^{2} / \lambda^{0}}$, with $m_{\Phi}$ being the mass of the complex scalar doublet $\Phi$, or, equivalently,

$$
v^{0}=\frac{2 m_{W}^{0}}{e^{0}} \sqrt{1-\left(\frac{m_{W}^{0}}{m_{Z}^{0}}\right)^{2}}
$$

in terms of basic parameters of the broken phase [9]. The linear term in the bare Higgs potential is then quenched and cannot serve as a tadpole counterterm, so that the tadpole contributions, which carry gauge dependence, need to be properly included order by order [9]. Upon $\overline{\mathrm{MS}}$ renormalization, taking Eq. (1) with the superscripts 0 dropped to be exact, $v(\mu)$ and all the basic parameters, including $\lambda(\mu)$, are manifestly gauge independent to all orders. Consequently, the twofold vacuum stability condition [5],

$$
\lambda\left(\mu^{\text {cri }}\right)=\beta_{\lambda}\left(\mu^{\text {cri }}\right)=0,
$$

which fixes a second minimum that is degenerate with the first one, has gauge-independent solutions for the critical ultrahigh scale $\mu^{\text {cri }}$ and one free basic parameter, which we take to be $M_{t}^{\text {cri }}$, the upper bound on the top-quark pole mass $M_{t}$, which is much less precisely known than $M_{H}$ [2]. For comparisons with the literature, we also determine the $M_{H}$ lower bound $M_{H}^{\text {cri }}$ sloppily using as input the mass parameter $M_{t}^{\mathrm{MC}}$ [2] that is extracted from experimental data using Monte Carlo event generators merely equipped with leading-order (LO) hard-scattering matrix elements. The results for $\mu^{\text {cri }}$ obtained together with $M_{t}^{\text {cri }}$ and $M_{H}^{\text {cri }}$ are denoted as $\mu_{t}^{\text {cri }}$ and $\mu_{H}^{\text {cri }}$, respectively. While the criticality condition in Eq. (2) carries a very simple physical meaning and is straightforward to solve numerically, it is slightly scheme dependent. To assess this scheme dependence, we compare the results for $\mu_{i}^{\text {cri }}$ and $M_{i}^{\text {cri }}$ with $i=t, H$ with those obtained applying the consistent approach of Ref. [20], in which $V_{\text {eff }}(H)$ is reorganized in powers of $\hbar$, so that its expansion coefficients are gauge independent at its extrema [21]. Specifically, this amounts to solving

$$
\begin{aligned}
\lambda= & \frac{1}{256 \pi^{2}}\left[\left(g^{2}+g^{\prime 2}\right)^{2}\left(1-3 \ln \frac{g^{2}+g^{\prime 2}}{4}\right)\right. \\
& \left.+2 g^{\prime 4}\left(1-3 \ln \frac{g^{\prime 2}}{4}\right)-48 y_{t}^{4}\left(1-\ln \frac{y_{t}^{2}}{4}\right)\right],
\end{aligned}
$$

which follows from $d V_{\text {eff }}^{\mathrm{LO}}\left(\tilde{\mu}^{\mathrm{cri}}\right) / d H=0$, for the minimum $H=\tilde{\mu}^{\text {cri }}$ of $V_{\text {eff }}^{\mathrm{LO}}(H)$ and requiring that, at next-to-leading order $(\mathrm{NLO}), \quad V_{\min }^{\mathrm{NLO}}=V_{\mathrm{eff}}^{\mathrm{LO}}\left(\tilde{\mu}^{\text {cri }}\right)+V_{\mathrm{eff}}^{\mathrm{NLO}}\left(\tilde{\mu}^{\text {cri }}\right) \geq 0$ for
$M_{t} \leq \tilde{M}_{t}^{\text {cri }}$ or $M_{H} \geq \tilde{M}_{H}^{\text {cri }}$, which is conveniently achieved in the Landau gauge [11].

We adopt the input values $G_{F}=1.1663787(6) \times$ $10^{-5} \mathrm{GeV}^{-2}, \alpha_{S}^{(5)}\left(M_{Z}\right)=0.1185(6), M_{W}=80.385(15) \mathrm{GeV}$, $M_{Z}=91.1876(21) \mathrm{GeV}, \quad M_{H}=125.7(4) \mathrm{GeV}, \quad M_{t}^{\mathrm{MC}}=$ 173.21(87) $\mathrm{GeV}$, and $M_{b}=4.78(6) \mathrm{GeV}$ from Ref. [2], evolve $\alpha_{s}^{(5)}(\mu)$ from $\mu=M_{Z}$ to the matching scale $\mu^{\mathrm{thr}}=$ $\xi M_{t}^{\mathrm{MC}}$ in the $n_{f}=5$ effective theory using coupled QCD $\times$ QED beta functions through four loops in QCD [7] and three loops in QED [22], and evaluate there the $\overline{\mathrm{MS}}$ couplings of the full SM from

$$
\begin{aligned}
g^{2}(\mu) & =2^{5 / 2} G_{F} M_{W}^{2}\left[1+\delta_{W}(\mu)\right], \\
g^{2}(\mu)+g^{2}(\mu) & =2^{5 / 2} G_{F} M_{Z}^{2}\left[1+\delta_{Z}(\mu)\right], \\
\lambda(\mu) & =2^{-1 / 2} G_{F} M_{H}^{2}\left[1+\delta_{H}(\mu)\right], \\
y_{f}(\mu) & =2^{3 / 4} G_{F}^{1 / 2} M_{f}\left[1+\delta_{f}(\mu)\right], \\
g_{s}^{2}(\mu) & =4 \pi \alpha_{s}^{(5)}(\mu)\left[1+\delta_{\alpha_{s}}(\mu)\right],
\end{aligned}
$$

including the appropriate terms of $O\left(\alpha^{n}\right)$ with $n=1,2$ [12,14], $O\left(\alpha \alpha_{s}\right)$ [5,12-14], and $O\left(\alpha_{s}^{n}\right)$ with $n=1,2,3,4$ [15-18]. The threshold corrections $\delta_{i}(\mu)$ in Eq. (4) are expressed in terms of the $\overline{\mathrm{MS}}$ couplings $\alpha(\mu)$ and $\alpha_{s}(\mu)$, and the pole masses $M_{i}$. To avoid the theoretical uncertainty due to the hadronic contributions to the relationship between $\alpha(\mu)$ and $\alpha_{\mathrm{Th}}$ [2], we replace the latter by $G_{F}$ in the set of basic parameters by extracting $\alpha(\mu)$ selfconsistently from the exact relationship $1 /[4 \pi \alpha(\mu)]=$ $1 / g^{2}(\mu)+1 / g^{\prime 2}(\mu)$ [14]. We stress that the $\overline{\mathrm{MS}}$ couplings in Eq. (4) are manifestly gauge independent and, thanks to partial tadpole cancellations, also finite in the limit $M_{H} \rightarrow 0[12,14]$. The pole masses $M_{t}$ and $M_{b}$ are subject to renormalon ambiguities of $O\left(\Lambda_{\mathrm{QCD}}\right)$, which, for $M_{t}$, are still small against the experimental error [2] and, for $M_{b}$, are inconsequential because of the smallness of $y_{b}(\mu)$. The use of $\overline{\mathrm{MS}}$ masses $m_{q}(\mu)$ would avoid renormalon ambiguities at the expense of introducing unscreened tadpole contributions to restore gauge independence [9], which coincidentally reduce the scheme dependence of $m_{t}(\mu)$ [23], but spoil the perturbative expansion for $m_{b}(\mu)$ [12]. For completeness, we also study the $\overline{\mathrm{MS}}$ mass parameter of the Higgs potential, $m^{2}(\mu)=-2 m_{\Phi}^{2}(\mu)=2 v^{2}(\mu) \lambda(\mu)$, using

$$
v^{2}(\mu)=2^{-1 / 2} G_{F}^{-1}[1+\Delta \bar{r}(\mu)],
$$

where $\Delta \bar{r}(\mu)$ to $O\left(\alpha^{n}\right)$ with $n=1,2$ and $O\left(\alpha \alpha_{s}\right)$ may be found in Ref. [14]. $\Delta \bar{r}(\mu)$ is gauge independent, but diverges for $M_{H} \rightarrow 0$ due to unscreened tadpole contributions. We estimate the theoretical uncertainties in the $\overline{\mathrm{MS}}$ parameters for $\xi=1$ due to unknown higher-order corrections by considering both scale variations and truncation errors. In the first case, we in turn put $\xi=1 / 2$ and 2 in Eq. (4), return to $\xi=1$ using the RG equations, and select the larger one of the two deviations thus generated. In the second case, we find the full set of $\overline{\mathrm{MS}}$ parameters for $\xi=1$, including besides those in Eq. (4) also $m_{i}(\mu)$ with $i=W, Z, H, f$ and $v(\mu)$, by self-consistently solving the system 
TABLE I. Coefficients in Eq. (6). The entries in the last row are given in units of GeV.

\begin{tabular}{|c|c|c|c|c|c|c|c|c|}
\hline$\underline{x}$ & $x_{0}$ & $\Delta x_{\alpha_{s}}$ & $\Delta x_{M_{W}}$ & $\Delta x_{M_{H}}$ & $\Delta x_{M_{t}}$ & $\beta_{x}$ & $\delta x_{\mu}$ & $\delta x_{\text {tru }}$ \\
\hline$g$ & 0.35838 & $-3.8 \times 10^{-6}$ & $-2.3 \times 10^{-4}$ & $-2.5 \times 10^{-6}$ & $+7.1 \times 10^{-5}$ & $+2.1 \times 10^{-3}$ & $8.5 \times 10^{-5}$ & $6.4 \times 10^{-4}$ \\
\hline$g^{\prime}$ & 0.64812 & $+8.5 \times 10^{-7}$ & $+1.2 \times 10^{-4}$ & $-6.6 \times 10^{-7}$ & $-9.8 \times 10^{-6}$ & $-5.2 \times 10^{-3}$ & $5.8 \times 10^{-5}$ & $1.0 \times 10^{-3}$ \\
\hline$g_{s}$ & 1.16540 & $+2.7 \times 10^{-3}$ & $+8.9 \times 10^{-8}$ & $+7.8 \times 10^{-8}$ & $-4.0 \times 10^{-5}$ & $-7.2 \times 10^{-2}$ & $5.6 \times 10^{-5}$ & $\cdots$ \\
\hline$y_{t}$ & 0.93517 & $-3.6 \times 10^{-4}$ & $-1.3 \times 10^{-7}$ & $-8.6 \times 10^{-6}$ & $+5.1 \times 10^{-3}$ & $-5.2 \times 10^{-2}$ & $8.0 \times 10^{-4}$ & $1.2 \times 10^{-3}$ \\
\hline$y_{b}$ & 0.01706 & $-5.7 \times 10^{-5}$ & $-5.1 \times 10^{-10}$ & $+1.3 \times 10^{-7}$ & $-2.4 \times 10^{-7}$ & $-9.2 \times 10^{-4}$ & $2.5 \times 10^{-4}$ & $1.1 \times 10^{-3}$ \\
\hline$\lambda$ & 0.12714 & $-6.2 \times 10^{-6}$ & $-4.2 \times 10^{-7}$ & $+8.2 \times 10^{-4}$ & $+6.4 \times 10^{-5}$ & $-2.0 \times 10^{-2}$ & $5.8 \times 10^{-4}$ & $5.5 \times 10^{-4}$ \\
\hline$m$ & 131.86 & $-2.6 \times 10^{-3}$ & $-4.4 \times 10^{-4}$ & $+3.8 \times 10^{-1}$ & $+1.2 \times 10^{-1}$ & +2.6 & $7.3 \times 10^{-1}$ & $4.1 \times 10^{-2}$ \\
\hline
\end{tabular}

of equations that express $G_{F}$ and $M_{i}$ entirely in terms of these parameters, so that unscreened tadpole contributions have to cancel numerically. We cast our results for $x=g, g^{\prime}, g_{s}, y_{t}, y_{b}$, $\lambda, m$ in the form

$$
\begin{aligned}
x(\mu)= & x_{0}+\Delta x_{\alpha_{s}} \frac{\alpha_{s}^{(5)}\left(M_{Z}\right)-\alpha_{s}^{(5), \exp }\left(M_{Z}\right)}{\Delta \alpha_{s}^{(5), \exp }\left(M_{Z}\right)} \\
& +\Delta x_{M_{W}} \frac{M_{W}-M_{W}^{\exp }}{\Delta M_{W}^{\exp }}+\Delta x_{M_{H}} \frac{M_{H}-M_{H}^{\exp }}{\Delta M_{H}^{\exp }} \\
& +\Delta x_{M_{t}} \frac{M_{t}-M_{t}^{\exp }}{\Delta M_{t}^{\exp }}+\beta_{x} \frac{\mu-\mu^{\mathrm{thr}}}{\mu^{\mathrm{thr}}} \pm \delta x_{\mu} \\
& \pm \delta x_{\mathrm{tru}},
\end{aligned}
$$

allowing for linear extrapolations in the least precisely known input parameters quoted above [2] and $\mu^{\text {thr }}$, which we disentangle from $M_{t}^{\exp }=M_{t}^{\mathrm{MC}}$, and list the coefficients in Table I.

We now in turn apply criterion (2) and the approach of Ref. [20] and write the resulting critical masses and associated scales $X=M_{i}^{\text {cri }}, \mu_{i}^{\text {cri }}, \tilde{M}_{i}^{\text {cri }}, \tilde{\mu}_{i}^{\text {cri }}$ with $i=t, H$ in the form

$$
\begin{aligned}
X= & X_{0}+\Delta X_{\alpha_{s}} \frac{\alpha_{s}^{(5)}\left(M_{Z}\right)-\alpha_{s}^{(5), \exp }\left(M_{Z}\right)}{\Delta \alpha_{s}^{(5), \exp }\left(M_{Z}\right)} \\
& +\Delta X_{M} \frac{M-M^{\exp }}{\Delta M^{\exp }} \pm \delta X_{\mathrm{par}}+\delta X_{\mu}^{ \pm} \pm \delta X_{\mathrm{tru}}
\end{aligned}
$$

where $M=M_{H}\left(M_{t}\right)$ if $i=t(H), \Delta X_{\alpha_{s}}$ and $\Delta X_{M}$ are the $1 \sigma$ errors due to $\alpha_{s}^{(5)}\left(M_{Z}\right)$ and $M$, respectively, $\delta X_{\text {par }}$ are the residual parametric errors combined in quadrature, $\delta X_{\mu}^{ \pm}$are the shifts due to the choices $\xi=2^{ \pm 1}$, and $\delta X_{\text {tru }}$ are the truncation errors induced by those in Table I. The coefficients in Eq. (7) are collected in Table II. $\tilde{M}_{t}^{\text {cri }}$ is $0.20 \mathrm{GeV}$ larger than $M_{t}^{\text {cri }}$, and $\tilde{M}_{H}^{\text {cri }}$ is $0.40 \mathrm{GeV}$ smaller than $M_{H}^{\text {cri }}$. These shifts reflect the scheme dependence. $\mu_{t}^{\text {cri }}$ and $\mu_{H}^{\text {cri fall }}$ slightly short of $M_{P}$, for which $\log _{10} M_{P}=19.086$, where the SM definitely ceases to be valid, while $\tilde{\mu}_{t}^{\text {cri }}$ and $\tilde{\mu}_{H}^{\text {cri lie }}$ appreciably beyond $M_{P}$, which is an inherent problem of Ref. [20] and was cured there by the ad hoc introduction of some new dimension-six operator. In the remainder of this Letter, we concentrate on the approach based on Eq. (2) [5].

To assess the significance of the higher-order corrections that were not yet included in Ref. [5], namely the full $O\left(\alpha^{2}\right)$ terms in $\delta_{i}(\mu)$ with $i=W, Z, H, q[12,14]$, the $O\left(\alpha \alpha_{s}\right)$ term in $\delta_{\alpha_{s}}(\mu)$ [13], and the $O\left(\alpha_{s}^{4}\right)$ terms in $\delta_{\alpha_{s}}(\mu)$ [17] and $\delta_{q}(\mu)$ [18], we switch them off one at a time. The resulting central values and scale dependencies of the critical parameters are also contained in Table II. The $O\left(\alpha^{2}\right)$ terms in $\delta_{i}(\mu)[12,14]$ shift $M_{t}^{\text {cri }}$ and $M_{H}^{\text {cri by }}-0.11$ and $+0.24 \mathrm{GeV}$, respectively, and reduce their scale uncertainties by almost a factor of 3 . On the other hand, the $O\left(\alpha_{s}^{4}\right)$ terms in $\delta_{q}(\mu)$ [18] produce larger and opposite shifts in $M_{t}^{\text {cri }}$ and $M_{H}^{\text {cri }}$, namely +0.20 and $-0.42 \mathrm{GeV}$, respectively, but merely reduce their scale uncertainties by less than $10 \%$. The $O\left(\alpha \alpha_{s}\right)$ [5] and $O\left(\alpha_{s}^{4}\right)$ [17] terms in $\delta_{\alpha_{s}}(\mu)$ are much less significant.

TABLE II. Coefficients in Eq. (7) and central values with scale dependencies obtained upon switching off the $O\left(\alpha^{2}\right)$ terms in $\delta_{i}(\mu)$ with $i=W, Z, H, q$, the $O\left(\alpha \alpha_{s}\right)$ and $O\left(\alpha_{s}^{4}\right)$ terms in $\delta_{\alpha_{s}}(\mu)$, and the $O\left(\alpha_{s}^{4}\right)$ terms in $\delta_{q}(\mu)$ one at a time. The unit of mass is taken to be $\mathrm{GeV}$.

\begin{tabular}{lccllcccccc}
\hline \hline$X$ & $X_{0}$ & $\Delta X_{\alpha_{s}}$ & $\Delta X_{M}$ & $\delta X_{\mathrm{par}}$ & $\delta X_{\mu}^{+}$ & $\delta X_{\mu}^{-}$ & $\delta X_{\text {tru }}$ & $\delta_{i}^{O\left(\alpha^{2}\right)}$ & $\delta_{\alpha_{s}}^{O\left(\alpha \alpha_{s}, \alpha_{s}^{4}\right)}$ \\
\hline$M_{t}^{\text {cri }}$ & 171.44 & 0.23 & 0.20 & 0.001 & -0.36 & 0.17 & -0.02 & $171.55_{+1.04}^{-0.47}$ & $171.43_{+0.17}^{-0.36}$ & $171.24_{+0.19}^{-0.38}$ \\
$\log _{10} \mu_{t}^{\text {cri }}$ & 17.752 & -0.051 & 0.083 & 0.007 & 0.007 & -0.006 & -0.002 & $17.783_{-0.008}^{+0.062}$ & $17.754_{-0.006}^{+0.007}$ & $17.751_{-0.007}^{+0.007}$ \\
$M_{H}^{\text {cri }}$ & 129.30 & -0.49 & 1.79 & 0.002 & 0.72 & -0.33 & 0.04 & $129.06_{-2.14}^{+0.95}$ & $129.32_{-0.33}^{+0.73}$ & $129.72_{-0.38}^{+0.76}$ \\
$\log _{10} \mu_{H}^{\text {cri }}$ & 18.512 & -0.158 & 0.381 & 0.008 & 0.173 & -0.082 & 0.008 & $18.495_{-0.531}^{+0.226}$ & $18.518_{-0.082}^{+0.174}$ & $18.602_{-0.094}^{+0.184}$ \\
$\tilde{M}_{t}^{\text {cri }}$ & 171.64 & 0.23 & 0.20 & 0.001 & -0.36 & 0.17 & -0.02 & $171.74_{+1.04}^{-0.46}$ & $171.63_{+0.17}^{-0.36}$ & $171.43_{+0.19}^{-0.37}$ \\
$\log _{10} \tilde{\mu}_{t}^{\text {cri }}$ & 21.442 & -0.059 & 0.094 & 0.005 & -0.083 & 0.022 & 0.002 & $21.485_{+0.343}^{-0.035}$ & $21.445_{+0.022}^{-0.083}$ & $21.441_{+0.014}^{-0.0072}$ \\
$\tilde{M}_{H}^{\text {cri }}$ & 128.90 & -0.49 & 1.79 & 0.003 & 0.73 & -0.34 & 0.04 & $128.67_{-2.15}^{+0.95}$ & $128.92_{-0.34}^{+0.73}$ & $129.32_{-0.38}^{+0.76}$ \\
$\log _{10} \tilde{\mu}_{H}^{\text {cri }}$ & 22.209 & -0.181 & 0.436 & 0.007 & 0.092 & -0.062 & 0.013 & $22.201_{-0.171}^{+0.146}$ & $22.217_{-0.062}^{+0.094}$ & $22.312_{-0.082}^{+0.1113}$ \\
\hline \hline
\end{tabular}




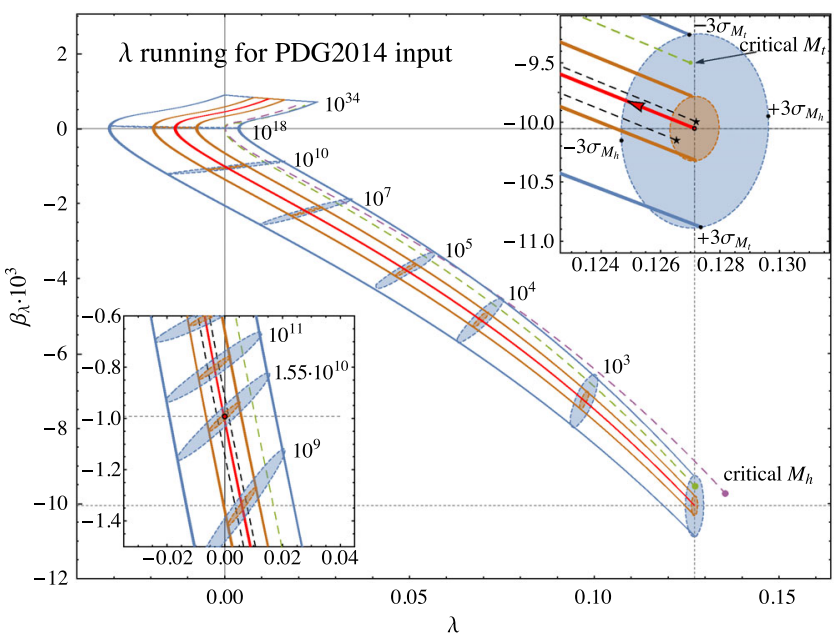

FIG. 1 (color online). RG evolution of $\lambda(\mu)$ from $\mu^{\text {thr }}$ to $\mu^{\text {cri }}$ and beyond in the $\left(\lambda, \beta_{\lambda}\right)$ plane for default input values and matching scale (red solid line), effects of $1 \sigma$ (brown solid lines) and $3 \sigma$ (blue solid lines) variation in $M_{t}^{\mathrm{MC}}$, theoretical uncertainty due to the variation of $\xi$ from $1 / 2$ to 2 (upper and lower black dashed lines with asterisks in the insets), and results for $M_{t}^{\text {cri }}$ (green dashed line) and $M_{H}^{\text {cri }}$ (purple dashed line). The $1 \sigma$ (brown ellipses) and $3 \sigma$ (blue ellipses) contours due to the errors in $M_{t}^{\mathrm{MC}}$ and $M_{H}$ are indicated for selected values of $\mu$. The insets in the upper right and lower left corners refer to $\mu=M_{t}^{\mathrm{MC}}$ and $\mu=1.55 \times 10^{10} \mathrm{GeV}$, respectively.

All these observations approximately carry over to $\tilde{M}_{t}^{\text {cri }}$ and $\tilde{M}_{H}^{\text {cri }}$.

Apart from the issue of gauge dependence, our analysis differs from that of Refs. $[10,11]$ in the following respects. In Refs. [10,11], the $O\left(\alpha \alpha_{s}\right)$ term in $\delta_{\alpha_{s}}(\mu)$ [13] and the $O\left(\alpha_{s}^{4}\right)$ terms in $\delta_{\alpha_{s}}(\mu)$ [17] and $\delta_{q}(\mu)$ [18] were not included; $\mu^{\text {thr }}$ was affected by the $M_{t}^{\mathrm{MC}}$ variation, which explains the sign difference in the corresponding shift in $M_{H}^{\text {cri }}$; and the scale uncertainties were found to be approximately half as large as here for reasons unknown to us.

In Fig. 1, the RG evolution flow from $\mu^{\text {thr }}$ to $\mu^{\text {cri }}$ and beyond is shown in the $\left(\lambda, \beta_{\lambda}\right)$ plane. The propagation with $\mu$ of the $1 \sigma$ and $3 \sigma$ confidence ellipses with respect to $M_{t}^{\mathrm{MC}}$ and $M_{H}$ tells us that the second condition in Eq. (2) is almost automatic, the ellipses for $\mu=10^{18} \mathrm{GeV}$ being approximately degenerated to horizontal lines. For default input values, $\lambda(\mu)$ crosses zero at $\mu=1.55 \times 10^{10} \mathrm{GeV}$. The contour of $M_{t}^{\text {cri }}$ approximately coincides with the right envelope of the $2 \sigma$ ellipses, while the one of $M_{H}^{\text {cri }}$, which relies on $M_{t}^{\mathrm{MC}}$, is driven outside the $3 \sigma$ band as $\mu$ runs from $\mu_{H}^{\text {cri }}$ to $\mu^{\text {thr }}$.

Our upgraded and updated version of the familiar phase diagram $[10,11,20,24]$ is presented in Fig. 2. Besides the boundary of the stable phase defined by Eq. (2), on which the critical points with $M_{t}^{\text {cri }}$ and $M_{H}^{\text {cri }}$ are located, we also show contours of $\lambda\left(\mu^{0}\right)=0$ and $\beta_{\lambda}\left(\mu^{0}\right)=0$. The demarcation line between the metastable phase and the instable one, in which the lifetime of our vacuum is shorter than the

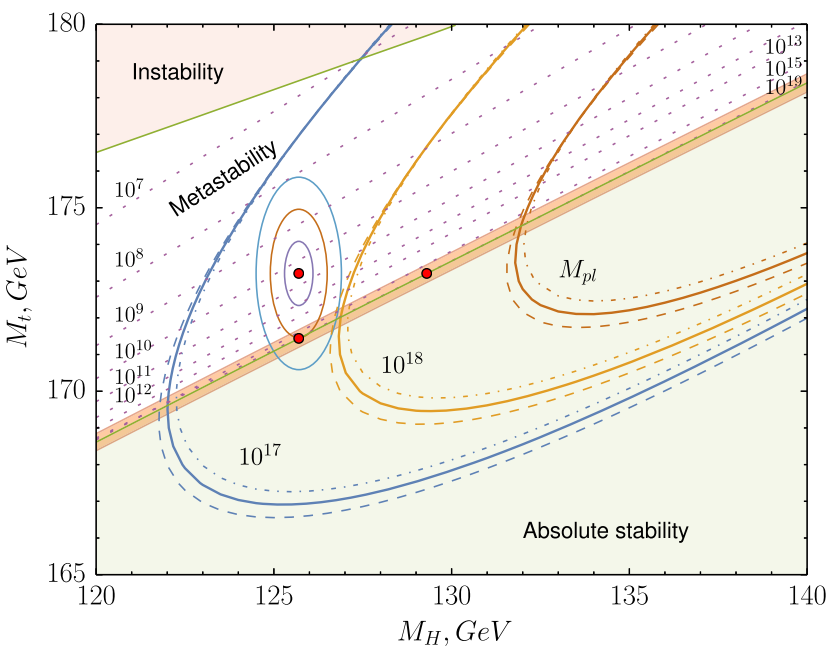

FIG. 2 (color online). Phase diagram of vacuum stability (lightgreen shaded area), metastability, and instability (pink shaded area) in the $\left(M_{H}, M_{t}\right)$ plane, contours of $\lambda\left(\mu^{0}\right)=0$ for selected values of $\mu^{0}$ (purple dotted lines), contours of $\beta_{\lambda}\left(\mu^{0}\right)=0$ for selected values of $\mu^{0}$ (solid parabolalike lines) with uncertainties due to $1 \sigma$ error in $\alpha_{S}^{(5)}\left(M_{Z}\right)$ (dashed and dot-dashed lines), critical line of Eq. (2) (solid green line) with uncertainty due to $1 \sigma$ error in $\alpha_{s}^{(5)}\left(M_{Z}\right)$ (orange shaded band), and critical points with $M_{t}^{\text {cri }}$ (lower red bullet) and $M_{H}^{\text {cri }}$ (right red bullet). The present world average of $\left(M_{t}^{\mathrm{MC}}, M_{H}\right)$ (upper left red bullet) and its $1 \sigma$ (purple ellipse), $2 \sigma$ (brown ellipse), and $3 \sigma$ (blue ellipse) contours are marked for reference.

age of the Universe, is evaluated as in Ref. [20] and represents the only gauge-dependent detail in Fig. 2. The customary confidence ellipses with respect to $M_{t}^{\mathrm{MC}}$ and $M_{H}$, which are included Fig. 2 for reference, have to be taken with caution because they misleadingly suggest that the tree-level mass parameter $M_{t}^{\mathrm{MC}}$ and its error [2] identically carry over to $M_{t}$, which is actually the real part of the complex pole position upon mass renormalization in the on-shell scheme [25]. In view of the resonance property, a shift of order $\Gamma_{t}=2.00 \mathrm{GeV}$ [2] would be plausible, which should serve as a useful error estimate for the time being.

In conclusion, we performed a high-precision analysis of the vacuum stability in the SM incorporating full two-loop threshold corrections [5,12-14], three-loop beta functions [6], and $O\left(\alpha_{s}^{4}\right)$ corrections to the matching and running of $g_{s}[7,17]$ and $y_{q}[8,18]$, and adopting two gauge-independent approaches, one based on the criticality criterion (2) for $\lambda(\mu)$ [5] and one on a reorganization of $V_{\text {eff }}(H)$ so that its minimum is gauge independent order by order [20]. For the $M_{t}$ upper bound we thus obtained $M_{t}^{\text {cri }}=(171.44 \pm$ $\left.0.30_{-0.36}^{+0.17}\right) \mathrm{GeV} \quad$ and $\tilde{M}_{t}^{\text {cri }}=\left(171.64 \pm 0.30_{-0.36}^{+0.17}\right) \mathrm{GeV}$, respectively, where the first errors are experimental, due the $1 \sigma$ variations in the input parameters [2], and the second ones are theoretical, due to the scale and truncation uncertainties. In want of more specific information, we assume the individual error sources to be independent and 
combine them quadratically to be on the conservative side. The $0.20 \mathrm{GeV}$ difference between the central values of $M_{t}^{\text {cri }}$ and $\tilde{M}_{t}^{\text {cri }}$ indicates the scheme dependence, which arguably comes as a third independent source of theoretical uncertainty. As our final result, we hence quote the combined value $\hat{M}_{t}^{\text {cri }}=\left(171.54 \pm 0.30_{-0.41}^{+0.26}\right) \mathrm{GeV}$, which is compatible with $M_{t}^{\mathrm{MC}}=(173.21 \pm 0.87) \mathrm{GeV}$ at the $1.3 \sigma$ level. In view of this and the present lack of knowledge of the precise relationship between and $M_{t}^{\mathrm{MC}}$ and $M_{t}$ mentioned above, the familiar notion $[10,11]$ that our vacuum is metastable is likely to be premature [24].

We thank W. Frost and M.D. Schwartz for useful correspondence regarding Ref. [20] and M. Yu. Kalmykov for critical comments. This work was supported in part by DFG Grant No. SFB 676, Ministry of Education and Science of the Russian FederationMES of Russia Grant No. MK-1001.2014.2, the Heisenberg-Landau Programme, and the Dynasty Foundation.

[1] G. Aad et al. (ATLAS Collaboration), Phys. Lett. B 716, 1 (2012); S. Chatrchyan et al. (CMS Collaboration), ibid. 716, 30 (2012).

[2] K. A. Olive et al. (Particle Data Group), Chin. Phys. C 38, 090001 (2014).

[3] N. V. Krasnikov, Yad. Fiz. 28, 549 (1978) [Sov. J. Nucl. Phys. 28, 279 (1978)]; H. D. Politzer and S. Wolfram, Phys. Lett. 82B, 242 (1979); 83B, 421(E) (1979); P. Q. Hung, Phys. Rev. Lett. 42, 873 (1979).

[4] E. Greenwood, E. Halstead, R. Poltis, and D. Stojkovic, Phys. Rev. D 79, 103003 (2009).

[5] F. Bezrukov, M. Yu. Kalmykov, B. A. Kniehl, and M. Shaposhnikov, J. High Energy Phys. 10 (2012) 140.

[6] L. N. Mihaila, J. Salomon, and M. Steinhauser, Phys. Rev. Lett. 108, 151602 (2012); Phys. Rev. D 86, 096008 (2012); K. G. Chetyrkin and M. F. Zoller, J. High Energy Phys. 06 (2012) 033; 04 (2013) 091; 09 (2013) 155(E); A. V. Bednyakov, A. F. Pikelner, and V. N. Velizhanin, ibid. 01 (2013) 017; Phys. Lett. B 722, 336 (2013); Nucl. Phys. B875, 552 (2013).

[7] T. van Ritbergen, J. A. M. Vermaseren, and S. A. Larin, Phys. Lett. B 400, 379 (1997); M. Czakon, Nucl. Phys. B710, 485 (2005).
[8] K. G. Chetyrkin, Phys. Lett. B 404, 161 (1997); Nucl. Phys. B710, 499 (2005); J. A. M. Vermaseren, S. A. Larin, and T. van Ritbergen, Phys. Lett. B 405, 327 (1997).

[9] R. Hempfling and B. A. Kniehl, Phys. Rev. D 51, 1386 (1995).

[10] G. Degrassi, S. Di Vita, J. Elias-Miró, J. R. Espinosa, G. F. Giudice, G. Isidori, and A. Strumia, J. High Energy Phys. 08 (2012) 098.

[11] D. Buttazzo, G. Degrassi, P. P. Giardino, G. F. Giudice, F. Sala, A. Salvio, and A. Strumia, J. High Energy Phys. 12 (2013) 089.

[12] B. A. Kniehl and O. L. Veretin, Nucl. Phys. B885, 459 (2014); B894, 56(E) (2015).

[13] A. V. Bednyakov, Phys. Lett. B 741, 262 (2015).

[14] B. A. Kniehl, A. F. Pikelner, and O. L. Veretin, Nucl. Phys. B896, 19 (2015).

[15] K. G. Chetyrkin, B. A. Kniehl, and M. Steinhauser, Phys. Rev. Lett. 79, 2184 (1997); Nucl. Phys. B510, 61 (1998).

[16] K. G. Chetyrkin and M. Steinhauser, Phys. Rev. Lett. 83, 4001 (1999); Nucl. Phys. B573, 617 (2000); K. Melnikov and T. van Ritbergen, Phys. Lett. B 482, 99 (2000).

[17] Y. Schröder and M. Steinhauser, J. High Energy Phys. 01 (2006) 051; K. G. Chetyrkin, J. H. Kühn, and C. Sturm, Nucl. Phys. B744, 121 (2006); B. A. Kniehl, A. V. Kotikov, A. I. Onishchenko, and O. L. Veretin, Phys. Rev. Lett. 97, 042001 (2006).

[18] P. Marquard, A. V. Smirnov, V. A. Smirnov, and M. Steinhauser, Phys. Rev. Lett. 114, 142002 (2015).

[19] L. Di Luzio and L. Mihaila, J. High Energy Phys. 06 (2014) 079.

[20] A. Andreassen, W. Frost, and M. D. Schwartz, Phys. Rev. Lett. 113, 241801 (2014); Phys. Rev. D 91, 016009 (2015).

[21] N. K. Nielsen, Nucl. Phys. B101, 173 (1975); R. Fukuda and T. Kugo, Phys. Rev. D 13, 3469 (1976).

[22] J. Erler, Phys. Rev. D 59, 054008 (1999).

[23] F. Jegerlehner, M. Yu. Kalmykov, and B. A. Kniehl, Phys. Lett. B 722, 123 (2013).

[24] S. Alekhin, A. Djouadi, and S. Moch, Phys. Lett. B 716, 214 (2012).

[25] B. A. Kniehl and A. Sirlin, Phys. Rev. D 77, 116012 (2008); 85, 036007 (2012); B. A. Kniehl, Phys. Rev. Lett. 112, 071603 (2014); Phys. Rev. D 89, 096005 (2014); 89, 116010 (2014). 